\title{
A radiation emission shielding method for high intensity focus ultrasound probes
}

\author{
Hao $\mathrm{Wu}^{\mathrm{a}, \mathrm{b}}$, Guofeng Shen ${ }^{\mathrm{a}, \mathrm{b},{ }^{*}}$ and Yazhu Chen ${ }^{\mathrm{a}, \mathrm{b}}$ \\ ${ }^{a}$ Biomedical Instrument Institute, School of Biomedical Engineering, Shanghai Jiao Tong University, \\ Shanghai 200030, P.R. China \\ ${ }^{b}$ Med-X Research Institute, Shanghai Jiao Tong University, Shanghai, 200030, P.R. China
}

\begin{abstract}
Electromagnetic compatibility (EMC) is a key issue in the design and development of safe and effective medical instruments. The treatment probes of high intensity focused ultrasound (HIFU) systems not only receive and transmit electromagnetic waves, but also radiate ultrasound waves, resulting in electromagnetic coupling. In this paper, an electromagnetic shielding method involving the enclosure of the probe in a copper wire mesh was introduced. First, sound pressure distribution simulations and measurements were performed using a hydrophone in order to evaluate the effects of the wire mesh on the acoustic performance of the HIFU system. The results indicated that the wire mesh did not disturb the normalized sound pressure field. In addition, the attenuation of the maximum pressure in the focal plane was equal to $6.2 \%$. Then, the electronic emission level was tested in a chamber. After the implementation of the wire mesh, the 10-100 $\mathrm{MHz}$ frequency band radiation was suppressed, and the HIFU system satisfied the national EMC standards.
\end{abstract}

Keywords: High intensity focused ultrasound, electromagnetic compatibility, radiated emission, radio frequency shielding

\section{Introduction}

High intensity focused ultrasound (HIFU) thermal ablation is a treatment that delivers a high-powered ultrasound beam to a predetermined target point (focus) in diseased tissue. The ultrasound energy is converted to thermal energy, which heats and ablates the tissue in the focal region while leaving the surrounding tissue unaffected. Since it is non-invasive, HIFU is a promising alternative to surgery for tumor treatment $[1,2]$.

However, the electromagnetic interference (EMI) resulting from the use of electronic instruments cannot be disregarded. The interference between medical equipment is particularly notable since it not only results in malfunctions, but also endangers the lives of patients [3]. China completed and implemented its medical electrical equipment electromagnetic compatibility standards in 2012 and 2014, respectively. According to these standards, since the safety and effectiveness of HIFU technology must be monitored, it belongs to the third category of medical instruments and must satisfy the EMI test.

\footnotetext{
* Address for correspondence: Guofeng Shen. Room 123, No. 3 Teaching Building (Med-X Institute) Shanghai Jiao Tong University, No. 1954, Huashan Road, Xuhui District, Shanghai 200030, P.R. China. Tel.: +86 21 62933209; Fax: +86 21 62932156; E-mail: shenguofeng@sjtu.edu.cn.
} 
The EMI test consists of the conduction emission (CE) test and radiation emission (RE) test. In the prototype stage, the detection of CE problems can usually prevent interference [4]. However, RE can occur anywhere in these systems, and cannot be entirely eliminated. During the printed circuit board (PCB) layout stage, unnecessary coupling must be prevented and the strength of the radiation source must be reduced. In the system design stage, filters must be added to all of the interfaces. Since these measures are usually inadequate, shielding is implemented to reduce the remaining RE. When MRI is used to guide HIFU surgery, interference to the imaging system is a major issue. In the past, engineers have primarily focused on promoting the immunity of the imaging systems $[5,6]$ rather than shielding radio frequency emission since electrically shielding the probe without changing its acoustic properties is difficult.

In this study, the shielding method involving the encapsulation of HIFU probes with wire mesh was evaluated. First, the sound pressure distribution of the HIFU probe with the wire mesh was simulated. Next, the acoustic pressure profile was measured using a hydrophone. Then, the RE frequency spectra of the well-shielded system and unshielded system were captured and compared.

\section{Electromagnetic shielding method}

Phased HIFU systems are primarily comprised of a computer, a phase signal generator, amplifiers, a treatment probe, and a long cable connected to the probe. Many modules in this system can become interference sources. For example, when the switch mode amplifiers are implemented, the harmonics of the switching signals create severe interference. These signals can be coupled with any of the modules. The computer, phased signal generator, and amplifiers are enclosed in a shielding box. Thus, electromagnetic coupling with the ground primarily occurs as a result of stray capacitance from the long cable. Furthermore, RE mainly leaks from the cable and the probe. MRI-guided HIFU systems can have cables that are meters long, causing emission in the very high frequency band (VHF band, 30-300 MHz). Coaxial cables with a shield can be used to connect a system to its probe. However, if the probe is not shielded effectively, the shielded cable remains open at one end. This portion of the cable becomes a sleeve antenna, which emits internal common disturbance. Although the gain of a sleeve antenna is smaller than that of a monopole of the same size (a cable without shielding), RE still occurs.

In this paper, the transducer array was comprised of 65 elements with radii of $5 \mathrm{~mm}$. The working frequency was equal to $1.36 \mathrm{MHz}$. The bottom and sides of the probe were enclosed in an electrical conductive shell, and the top was covered with a copper wire mesh. The wire mesh electrically

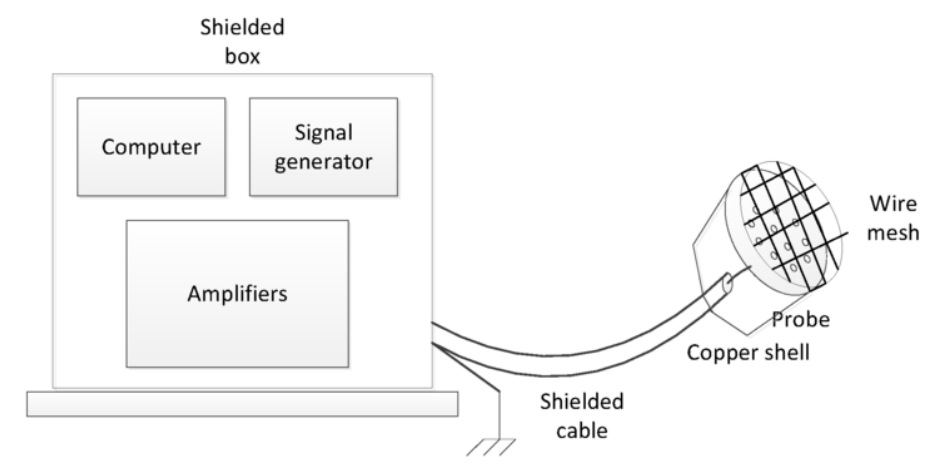

Fig. 1. System shielding structure. 
connected the shell and shielding layer of the cable. The wire mesh was curved to match the curvature of the spherical transducer array on the probe. The wire mesh was immerged in water; thus, the electromagnetic and acoustic media were water. The shielding structure of this system is depicted in Figure 1.

The wire radius and mesh aperture of wire mesh can significantly affect its acoustic transmittance and electromagnetic shielding effectiveness (SE). The wire radius of mesh determines its acoustic scattering power and angle [7]. When the wire radius is significantly smaller than the wavelength, the scattering power is small and the scattering angle is uniformly opposite of the incident direction. As the wire radius increases, the scattering power increases, and the scattering angle becomes complex and moves toward the incident direction. Scattering waves in the incident direction correspond with and can offset incident waves. The mesh aperture determines the number of scatters, and small apertures result in severe coupling among scatters. However, wire mesh with small mesh apertures possesses high SE values in certain frequency bands $[8,9]$. For the purposes of this paper, a $1.066 \mathrm{~mm}$ mesh aperture and $0.1 \mathrm{~mm}$ wire radius were used in order to optimize the SE and acoustic transmittance.

\section{Acoustic simulations}

The acoustic simulations involved a near field (near the transducer and wire mesh) and far field (far from the wire mesh). The near field was complex and the far field was simple. Therefore, a hybrid simulation method was used in order to reduce the computational time and increase the precision of the simulations. A numerical method [10] (the k-space method) was applied to the near field, and an analytical method [11] [a displaced point source method (DPSM)] was applied to the far field. An imaginary interface was artificially defined $7 \mathrm{~mm}$ behind the wire mesh to divide the near and far fields.

In the k-space method, the spatial differential operator in the wave equation is solved using Fourier transform and the time differential operator is solved using finite difference. After discretization, the sound speed and density of the voxels, which were occupied by the wire mesh, were set into much larger values than those of water in order to simulate rigid scattering. The computations were performed using k-Wave, a free Matlab toolkit available on www.k-wave.org. The particle velocities on the imaginary interface in three dimensions were obtained using the k-space method.

In DPSM, the real sound source is considered to be the superposition of the sound fields generated from the point sources artificially displaced on (or near) the surface of the actual source. Since the point sources are excited simultaneously, the sound pressure at point $\mathbf{x}$ can be obtained by integrating the acoustic waves of all of the sound points. The discretization of this integration can be expressed as

$$
p(\mathbf{x})=\sum_{m} A_{m} \frac{\exp \left(i k r_{m}\right)}{r_{m}}
$$

where $A_{m}$ is proportional to the velocity amplitude and area of the $m^{\text {th }}$ source point, which can be defined as the strength of the point sources; $k$ is the wave number; and $r_{m}$ is the distance between the $m^{\text {th }}$ source point and the target point. In this paper, the "real" source was the imaginary interface. The "strength" values of the point sources were calculated by reverse solving Eq. (1) and matching the particle velocities generated by the point sources on the imaginary interface to those obtained using 
the k-space method. The sound pressure at any point in the space could be calculated by forward solving Eq. (1).

Because the spaces of any two elements in the near field were identical, only one of the elements' particle velocity profiles was simulated. In order to generate a sheered focus, the phase differences between the elements were implied using the particle velocity profile obtained through the k-space method. The total sound pressure profile was obtained by summing the sound pressures of all of the elements from point to point. The phase differences were calculated using traditional methodology, and no phase corrections were applied.

In the simulations, the coordinate origin $[(0,0,0) \mathrm{mm}]$ was defined as the geometric focus of the transducer. The $z$-axis of the coordinate system extended from the transducer to the focus along the axis of the transducer. The $x y$-plane was the focal plane. The focus pressure was defined as $0.67 \mathrm{MPa}$ according to the measured results and this pressure was used in the RE test. The sound pressure profiles with focus at $(0,0,0) \mathrm{mm}$ and $(5,0,0) \mathrm{mm}$ were simulated respectively, as shown in Figure 2. The simulation results indicated that the wire mesh did not affect the sound pressure distribution. However, after the wire mesh was implemented, the maximum pressures on the focal plane reduced by $8.6 \%$ and $8.2 \%$ with focusing at $(0,0,0)$ and $(5,0,0)$, respectively.

The three-dimensional $\mathrm{k}$-space simulation was time-consuming since it involved computing the sound field for the entire space in every time step. This computational time could be reduced by decreasing the spatial and time resolution. However, in order to maintain the stability of the simulation, the Courant-Friedrichs-Lewy (CFL) number would need to satisfy [12].

$$
C F L \equiv \frac{c_{0} \Delta t}{\Delta x} \leq \frac{2}{\pi} \frac{c_{0}}{c_{\max }}
$$

where $c_{0}$ is the sound speed of water, $c_{\max }$ is the sound speed of the wire, $\Delta t$ is the time step, and $\Delta x$ is the spatial step. Thus, a high relative sound speed $\left(c_{\max } / c_{0}\right)$, high special resolution, and short computational time could not be achieved simultaneously. A small spatial step and a high relative sound speed (representing high reflectivity) could be used to accurately describe the geometry and acoustic characteristics of the wire mesh, respectively. In this paper, in order to optimize the computational speed and accuracy of the simulations, the relative sound speed, time step, and space step were defined as $20,0.99 \mathrm{~ns}$, and $0.06 \mathrm{~mm}$, respectively.

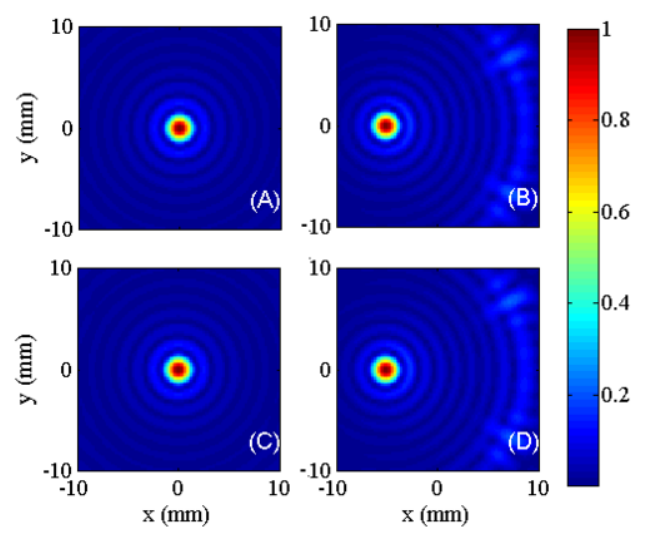

Fig. 2. Normalized sound pressure profiles in the free field (A and B) on the focal plane and $(C$ and $D)$ with the wire mesh. The foci of $(A)$ and $(C)$ and $(B)$ and $(D)$ were located at $(0,0,0) \mathrm{mm}$ and $(5,0,0) \mathrm{mm}$, respectively. 


\section{Experimental measurements}

The transducer probe was mounted to the bottom of a polymethyl cubic tank filled with degassed water. Then, the acoustic power was measured. The length of side of the tank, which was much larger than the aperture diameter of the transducer array, was equal to $400 \mathrm{~mm}$. Therefore, the boundary of the sound field was assumed to be infinite. The sound field boundary was simulated in the k-space with a perfectly matched layer (PML). The sound pressure profiles were record using a hydrophone. At each position, the virtual value of pressure was measured 5 times, and the average value was recorded. The pressure profiles on the focal plane when the focus was steered to the geometric center of the array are present in Figure 3. The normalized pressure profiles with and without the wire mesh in the propagation path of the acoustic waves were almost identical. With the same phase calculation algorithm, no additional side lobes were generated as a result of the wire mesh.

The acoustic power was measured using a radiation force balance (RFB) power meter in order to detect any reductions in power due to the wire mesh. The focus was steered to the geometric center in order to ensure maximum acoustic power. The results are shown in Figure 4. In this figure, U2/U2max is the squared driven voltage ratio, which represents the amount of output acoustic power. The power decreased by approximately $11 \%$ and did not vary with the acoustic power. The measurement results indicated that the wire mesh did not disturb the sound field when the wire diameter was less than or equal to the wave length.

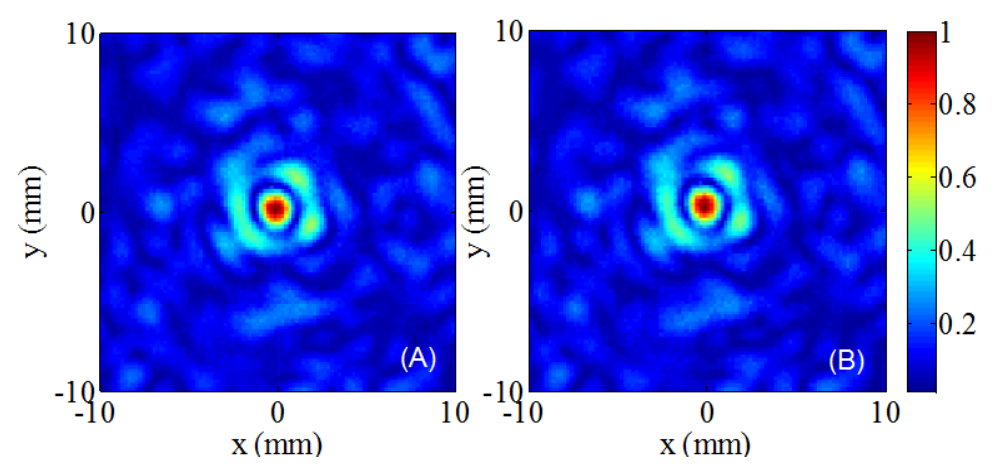

Fig. 3. Normalized sound pressure profiles (A) without and (B) with the wire mesh.

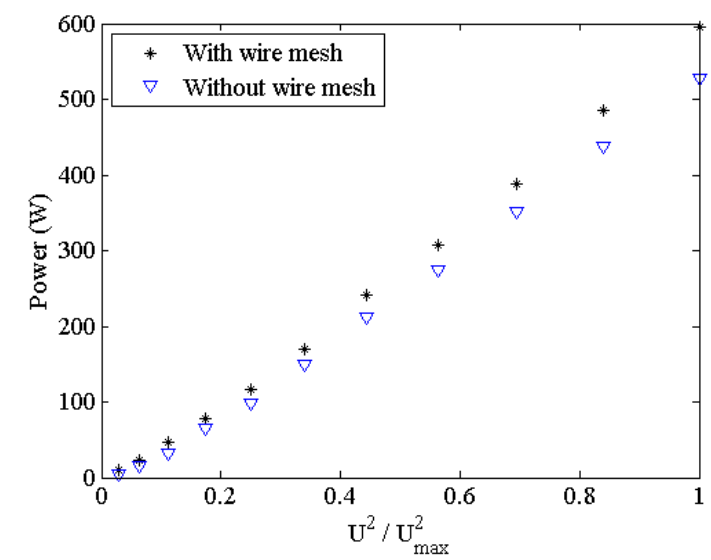

Fig. 4. Acoustic power with and without the wire mesh. 


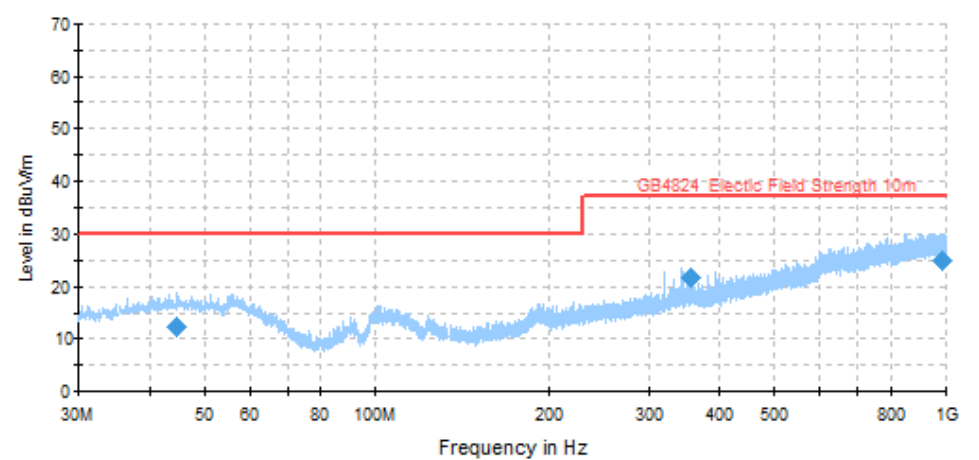

(A) Background radiation in the chamber.

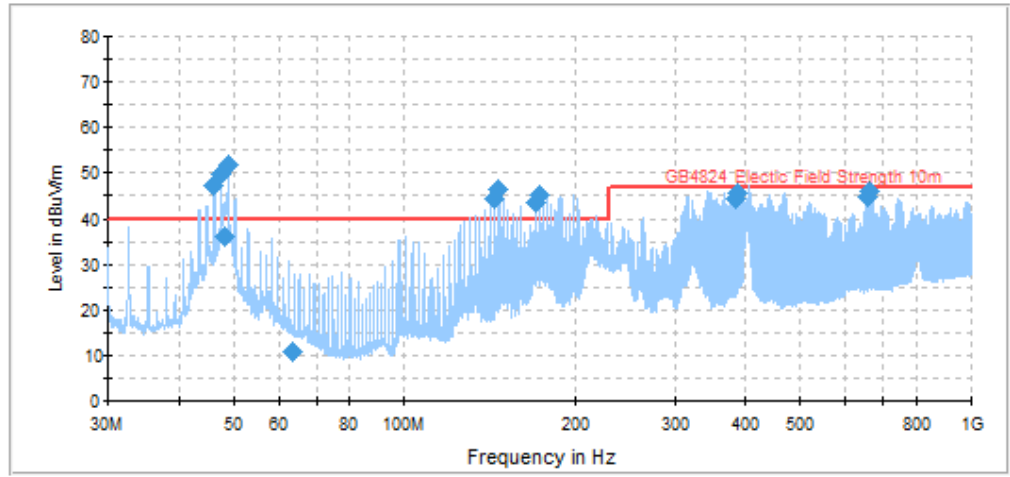

(B) Without the wire mesh.

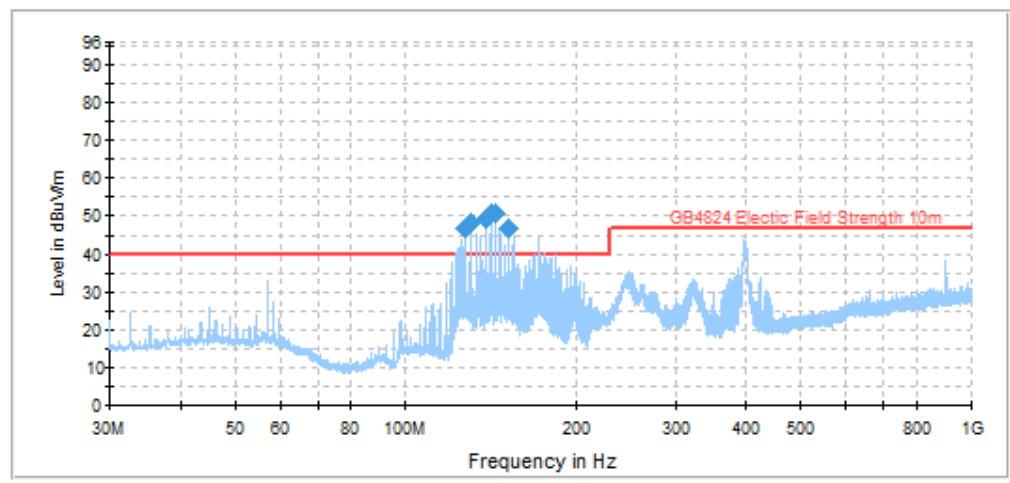

(C) With the wire mesh.

Fig. 5. EMC testing frequency spectra.

The RE test was performed in an electromagnetic anechoic chamber. The testing platform in the chamber consisted of a receiving antenna and a rotating table. The antenna was capable of moving up and down and varying between horizontal and vertical polarization. The HIFU system with the probe and the polymethyl tank was placed on the rotating table so that the radiation emitted from different angles could be record by the antenna. In this test, the antenna was initially defined at $1 \mathrm{~m}$ with horizontal polarization. Each time the antenna rose by $1 \mathrm{~m}$, the equipment under test (EUT) rotated $180^{\circ}$, during which the maximum emission level was record. When the test was completed with the 
antenna at $4 \mathrm{~m}$, the antenna switched to vertical polarization. Then, it moved downward and the testing procedure was repeated. The maximum values at all of the frequency points were collected, as shown in Figure 5. Compared to the background radiation in the chamber shown in Figure 5(A), the noise in the $40-60 \mathrm{MHz}$ band shown in (A) and the impulses appearing every $1.36 \mathrm{MHz}$ in the entire spectra shown in (B) and (C) were noise introduced by the HIFU system. The wire mesh effectively reduced the radiation emission, especially in the VHF band. When the probe was open, the emission levels exceeded the national standards (the red lines shown in the figures) by more than $10 \mathrm{~dB}$. After the wire mesh was mounted, the harmonic components observed as impulses were effectively reduced, and the emission levels satisfied the national standard in the VHF band. In the $100-200 \mathrm{MHz}$ frequency band, the emission levels were still high and exceeded the national standard. This could have been the result of other antennas with different emission bands, e.g., single-end-open communication cables with weak shielding.

\section{Conclusions}

In this paper, a wire mesh shielding method for HIFU probes was analyzed through simulations and experiments. The ultrasound probe was completely shielded from EMI after the implementation of the wire mesh. The RE levels were obviously reduced. The focusing capacity of the acoustic performance was not affected by the wire mesh in the acoustic propagation path. The acoustic power was attenuated, but not significantly. This method could be easily implemented and would not require any additional phase correction.

\section{Acknowledgments}

This study was supported by the National Natural Science Foundation of China (No. 30800246), the Key Technologies R \& D Program of Shanghai, China (No. 09441900500), and the Industry, Education, and Academy Project of the Shanghai Education Commission (No. 14cxy05).

\section{References}

[1] S. Crouzet, O. Rouviere and X. Martin, High-intensity focused ultrasound as focal therapy of prostate cancer, Current Point in Urology 24 (2014), 225-230.

[2] S. Li and P.H. Wu, Magnetic resonance image-guided versus ultrasound-guided high-intensity focused ultrasound in the treatment of breast cancer, Chinese Journal of Cancer 32 (2013), 441-452.

[3] M. Fernández Chimeno, M. Quílez and F. Silva, Understanding electrosurgical unit perturbations in order to address hospital operating room electromagnetic compatibility, IEEE Transactions on Biomedical Engineering 53 (2006), 1206-1209.

[4] M. I. Buzdugan, T. I. Buzdugan and H. Balan, Considerations on electromagnetic compatibility for medical devices, International Conference on Advancements of Medicine and Health Care through Technology IFMBE Proceedings 36 (2011), 94-99.

[5] Siemens Aktiengesellschaft, HIFU-Compatible Reception Coil for MRI RF Signals and Signal Receiving Method, 2008, US patent: US7463030B2.

[6] Siemens Aktiengesellschaft, Method and Apparatus for Reducing Aliasing Artifacts in the Imaging for MR-Monitored HIFU Therapy, 2010, US patent: US7759937B2.

[7] James J. Faran Jr., Sound scattering by solid cylinders and spheres, Journal of the Acoustical Society of America 23 (1951), 405-418. 
[8] L.F. Liu and Q.S. Zhang, Analysis of electromagnetic shielding effectiveness of metal material, Advanced Materials Research 538 (2012), 655-659.

[9] K.F. Casey, Electromagnetic shielding behavior of wire-mesh screens, IEEE Transactions on Electromagnetic Compatibility 30 (1988), 298-306.

[10] M. Tabei, T. D. Mast and R.C. Waag, A k-space method for coupled first-order acoustic propagation equations, The Journal of the Acoustical Society of America 111 (2002), 53-63.

[11] R. Ahmad, T. Kundu and D. Placko, Modeling of phased array transducers, The Journal of the Acoustical Society of America 117 (2005), 1762-1776.

[12] T.D. Mast, et al., A k-space method for large-scale models of wave propagation in tissue, IEEE Transactions on Ultrasonics, Ferroelectrics and Frequency Control 48 (2001), 341-354. 